\title{
A Total Synthesis of the Marine Alkaloid Discoipyrrole D
}

Yiwen Zhang and Martin G. Banwell*

Research School of Chemistry, Institute of Advanced Studies

The Australian National University, Canberra, ACT 2601, Australia

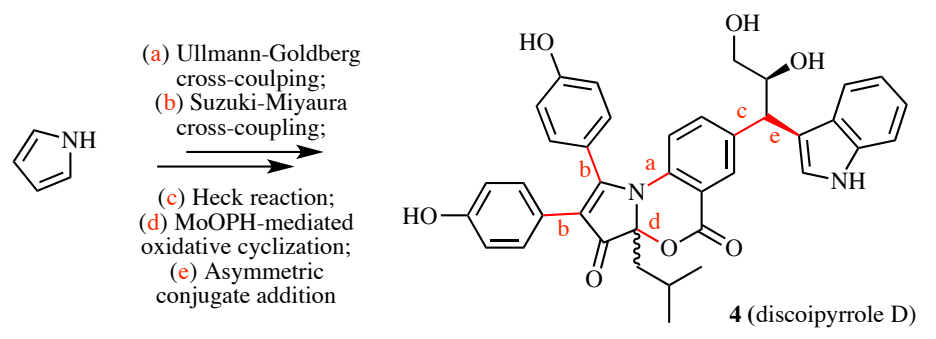

\begin{abstract}
A total synthesis of the diastereoisomeric pair of compounds, 4, assigned to the marine alkaloid discoipyrrole D is reported. A series of palladium-catalysed crosscoupling and other reactions was employed to assemble the relevant 1,2,3,4-tetra-substituted pyrrole (16) that was engaged in MoOPH-mediated oxidative cyclization then conjugate addition and redox processes to complete the synthesis. This work serves to confirm the structure (4) originally assigned to discoipyrrole D.
\end{abstract}




\section{INTRODUCTION}

In 2013 MacMillan and co-workers reported on the isolation (from a marine bacterium) and structural elucidation of the alkaloids 1-4, named discoipyrroles A-D respectively. ${ }^{1}$ The occurrence of these compounds as racemic or (in the case of compound 4) diastereoisomeric mixtures suggested that they were produced by non-enzymatic means and the same group was able to mimic the proposed biogenesis in a one-pot total synthesis of compound $\mathbf{1}$ by "incubating" a DMSO solution of $p$-hydroxysattabacin, $p$-hydroxybenzalehyde and anthranilic acid at $50{ }^{\circ} \mathrm{C}$ in the presence of $1 \%$ trifluoroacetic acid. ${ }^{1,2}$ The generation of analogues of the discoipyrroles by related means could well lead to the identification of compounds that show even more potent inhibition of the DDR signalling pathway than the natural products themselves. In a similar vein, May and co-workers were able to produce a brominated analogue of discoipyrrole A bis- $O$-methyl ether and elaborate this, through Hecktype chemistry followed by, inter alia, an organocatalyzed asymmetric conjugate addition of an indole trifluoroborate, to discoipyrrole D bis- $O$-methyl ether. ${ }^{3}$

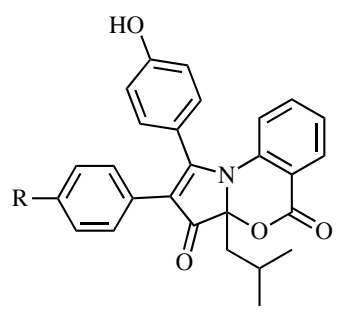

$\mathbf{1}(\mathrm{R}=\mathrm{OH}$, discoipyrrole $\mathrm{A})$ $2(\mathrm{R}=\mathrm{H}$, discoipyrrole $\mathrm{B})$

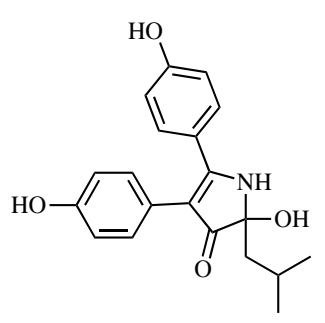

3 (discoipyrrole C)

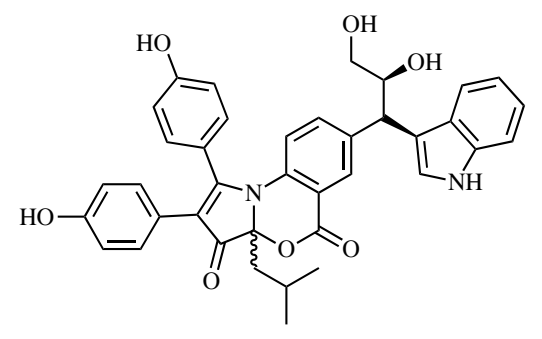

4 (discoipyrrole D)

Figure 1: Discoipyrroles A-D (1-4, respectively). 
Seemingly, however, the sensitivity of the $3 H$-benzo[ $d]$ pyrrole[1,3]oxazine-3,5-dione core associated with this last compound to the conditions normally used to cleave aryl methyl ethers prevented the completion of a synthesis of the natural product, viz. discoipyrrole D. ${ }^{3}$

In 2016 we reported ${ }^{4}$ a distinctly different route to compounds $\mathbf{1}$ and $\mathbf{2}$ that involved the assembly, through Ullmann-Goldberg ${ }^{5}$ and Suzuki-Miyaura cross-coupling chemistries, ${ }^{6}$ of a tetra-substituted pyrrole that could be engaged in an oxoperoxymolybdenum (pyridine) hexamethylphosphoric triamide ${ }^{7}$ or $\mathrm{MoOPH}$-mediated oxidative cyclisation reaction ${ }^{8}$ resulting in the assembly of the $3 H$-benzo $[d]$ pyrrole[1,3]oxazine-3,5-dione core of discoipyrroles A and B. We have since used this modular approach to the discoipyrroles in the construction of a range of analogues. ${ }^{9}$ As an extension of such work we now report on the amalgamation this chemistry with certain aspects of May's approach ${ }^{3}$ to discoipyrrole D in the successful synthesis of this natural product that serves to confirm the structure originally assigned to it by MacMillan and co-workers. ${ }^{1}$

\section{RESULTS AND DISCUSSION}

The two major considerations associated with our devising an approach to discoipyrrole D were, (i), the nature of the protecting group to be used to mask the phenolic hydroxyl groups present in the target and, (ii), the timing of MoOPH-mediated oxidative cyclisation reaction that establishes the $3 H$-benzo[d]pyrrole[1,3]oxazine-3,5-dione core associated with compound 4. After some preliminary experimentation we elected to employ the MOM group to protect the phenolic hydroxyls and to also delay the key oxidative cyclisation reaction as 
long as possible because of the seemingly "fragile" nature of the core heterocyclic ring system associated with the discoipyrroles. ${ }^{3}$

The reaction sequence leading to the tetrasubstituted pyrrole that was to be engaged in the pivotal oxidative cyclization reaction is shown in Scheme 1 and started with the regioselective Ullmann-Goldberg arylation of the parent heterocycle 5 with methyl 5-bromo2-iodobenzoate (6) under conditions reported by Buchwald and co-workers. ${ }^{5}$ Product 7 $(99 \%)$ obtained by this means was subjected to a Vilsmeier-Haack reaction ${ }^{10}$ and thus affording the pyrrole-2-carboxaldehyde $\mathbf{8}(83 \%)$ that was itself subjected to a regiocontrolled di-iodination reaction using molecular iodine in the presence of silver trifluoroacetate and thereby producing compound 9 (77\%). Two-fold Suzuki-Miyaura cross coupling of di-iodide 9 with commercially available $p$-hydroxyphenylboronic acid (10) then gave the tri-arylated pyrrole $11(84 \%)$ that was protected, under conventional conditions, as the corresponding bis-MOM ether 12 (99\%). Witting olefination of the aldehyde residue associated with the last compound using in situ generated isopropylenetriphenylphosphorane. ${ }^{11}$ By such means the olefin 13 (79\%) was obtained and this was immediately hydrogenated using dihydrogen in the presence of Adam's catalyst and so affording the isobutylated pyrrole $14(99 \%)$ that was itself engaged in a Heck reaction with 3,3-diethoxyprop-1-ene using $\mathrm{Pd}(\mathrm{OAc})_{2}$ as the catalyst source. The ester residue associated with product $15(65 \%)$ was saponified using sodium hydroxide in methanol and upon acidification of the ensuing mixture with aqueous $\mathrm{HCl}$ so as to generate the free acid the acetal moiety was also hydrolyzed and thus affording the cinnamaldehyde 16 (99\%), the substrate required for the pivotal oxidation reaction. All the 
spectral data acquired on this tetrasubstituted pyrrole were in complete accord with the illustrated structure.

Scheme 1: Synthesis of pyrrole 16 required for the pivotal oxidative cyclisation reaction.
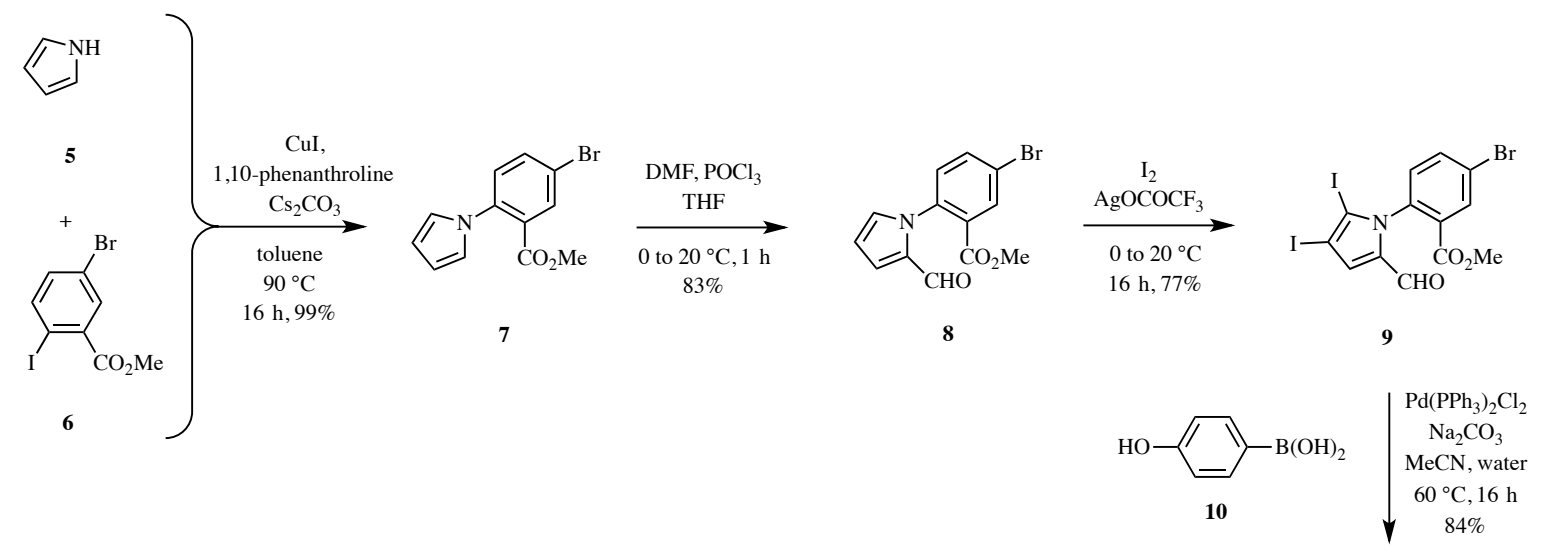

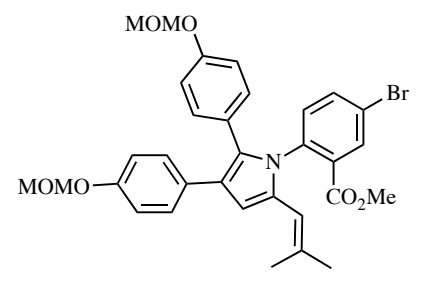

13
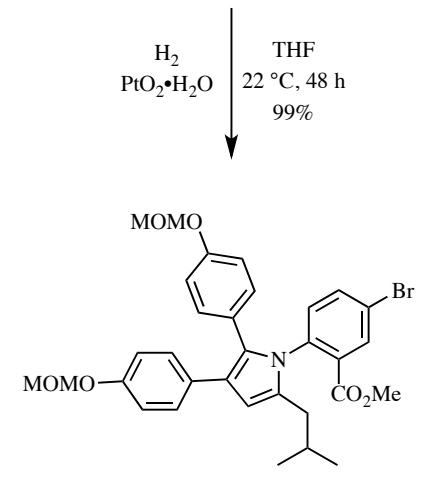

14

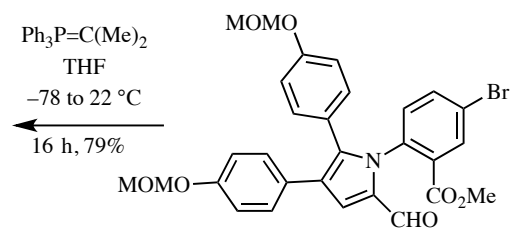

12

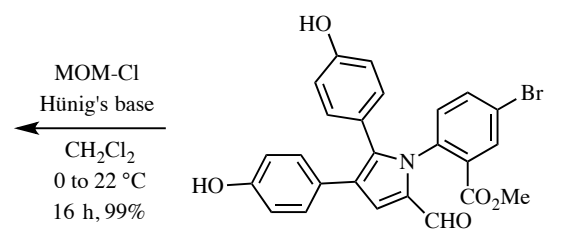

11
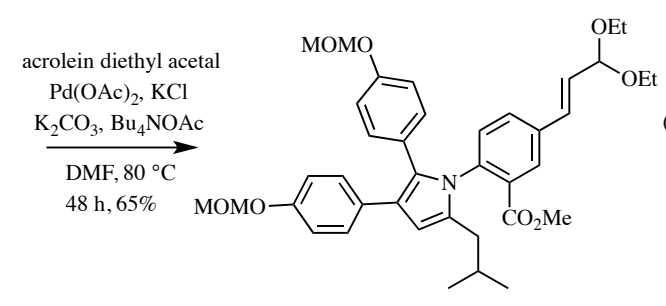

15

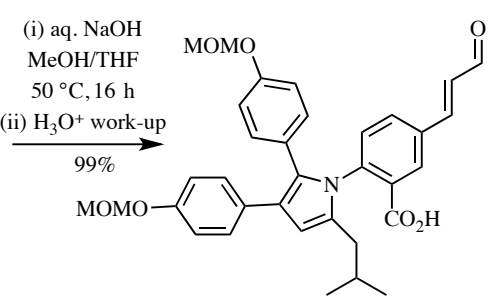

16

In keeping with earlier observations, ${ }^{4}$ when compound 16 was treated with freshly prepared MoOPH in methanol at ambient temperatures the anticipated oxidative cyclization reaction took place and after chromatographic purification of the major reaction product compound $\mathbf{1 7}$ 
was obtained in $50 \%$ yield as a yellow oil. No other characterizable materials could be isolated from the reaction mixture. The ${ }^{13} \mathrm{C}$ NMR spectrum of compound $\mathbf{1 7}$ displayed twenty-nine of the expected thirty resonances including three at $\delta_{C} 193.8,193.2$ and 167.4 ppm that are attributed to carbonyl carbons associated with the ketone, aldehyde and lactone residues, respectively. In the corresponding infra-red spectrum $\mathrm{C}=\mathrm{O}$ stretching bands are evident at 1740, 1702 and $1679 \mathrm{~cm}^{-1}$ while molecular associated ions at $m / z 606\left[(\mathrm{M}+\mathrm{Na})^{+}\right]$ and $584\left[(\mathrm{M}+\mathrm{H})^{+}\right]$dominate the EI mass spectrum.

Scheme 2: MoOPH-mediated oxidative cyclization of pyrrole 16 leading to the formation of $3 H$-benzo[ $d]$ pyrrole[1,3] oxazine-3,5-dione $\mathbf{1 7}$

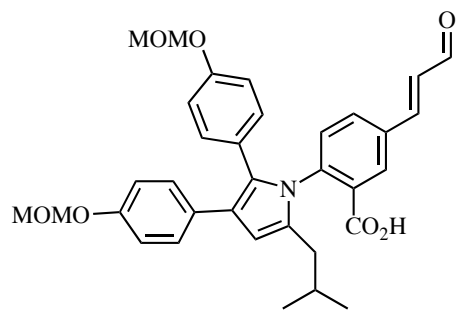

16

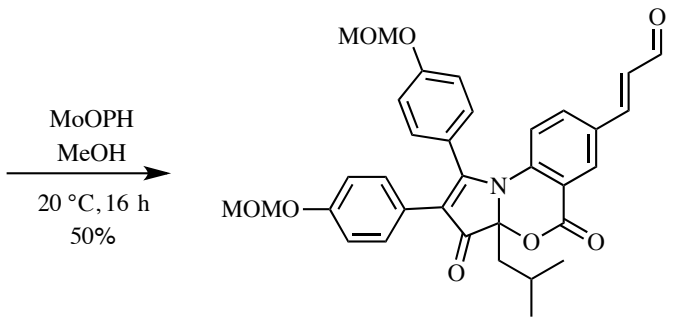

17

The completion of the synthesis of target compound 4 is shown in Scheme 3 and proved to be a rather straightforward matter. Thus, following the protocols reported May and coworkers, ${ }^{3}$ compound $\mathbf{1 7}$ was subjected to a reaction with the readily prepared and $\mathrm{N}$-Bocprotected indole $\mathrm{C} 3$-trifluorborate salt $\mathbf{1 8}$ in the presence of the freshly prepared catalyst $(R)$ $3,3^{\prime}-\left(\mathrm{C}_{7} \mathrm{~F}_{7}\right)_{2}-\mathrm{BINOL}^{3}$ and so affording product 19 as a 1:1 mixture of diastereoisomers. This outcome clearly indicates that the existing stereogenic center within substrate $\mathbf{1 7}$ has no impact on the configuration of $\mathrm{C} 1$ established during the conjugate addition reaction. By analogy with the work of May, this addition reaction is presumed to have proceeded with a 
high degree of stereochemical control and such that the illustrated $S$-configuration has been established at the new stereogenic center. May's three-step protocol was then employed to manipulate the indole-bearing carbon side-chain of compound 19 so as introduce the associated hydroxyl groups. Specifically, then, a $D$-proline-controlled oxidation involving nitrosobenzene $\mathrm{e}^{12}$ was used to introduce a $2^{\circ}$-phenyaminoxy moiety in a stereocontrolled fashion and this was followed by reduction of the aldehyde moiety using sodium triacetoxyborohydride. Treatment of the ensuing 2-aminoxyalcohol with nitrosobenzene resulted in cleavage of the aminoxy residue and so producing diol $\mathbf{2 0}(36 \%)$ that was also obtained as a 1:1 mixture of diastereoisomers. In the final steps of the reaction sequence, compound $\mathbf{2 0}$ was treated with trifluoroacetic acid (to cleave to Boc group) and then aqueous $\mathrm{HCl}$ (to cleave the MOM ethers) and thereby affording compound $\mathbf{4}$ in $60 \%$ yield and as a light-yellow oil.

All the spectral data acquired on compound $\mathbf{4}$, which indicated that it had been generated as a 1:1 mixture of diastereoisomers, proved a good match for those reported by MacMillan and co-workers ${ }^{1}$ on discoipyrrole D (see SI for a tabulated comparison of the ${ }^{13} \mathrm{C}$ NMR spectral data sets). Within the ${ }^{1} \mathrm{H}$ and ${ }^{13} \mathrm{C}$ NMR spectra of the synthetically derived material the presence of aliphatic impurities was evident but the aromatic regions of each spectrum were very clean. The origins of these impurities probably reflect the fragile nature of the $3 \mathrm{H}$ benzo $[d]$ pyrrole[1,3]oxazine-3,5-dione core of the compound and its partial degradation under the acidic conditions necessarily employed in the final steps of the synthesis. The synthetic material was optically active $\left\{[\alpha]_{\mathrm{D}}^{25}=+25(c 0.2, \mathrm{MeOH})\right\}$ and thus indicating that there had been some level of asymmetric induction in the conversion $17+18 \rightarrow 19$ and that a 
certain degree of stereochemical integrity was maintained over the remaining two steps of the synthesis. However, in the absence of any published specific rotation data on the title natural product not much more can be said about the absolute configuration and enantiomeric purity of the alkaloid.

Scheme 3: Completion of the synthesis of discoipyrrole D (4)
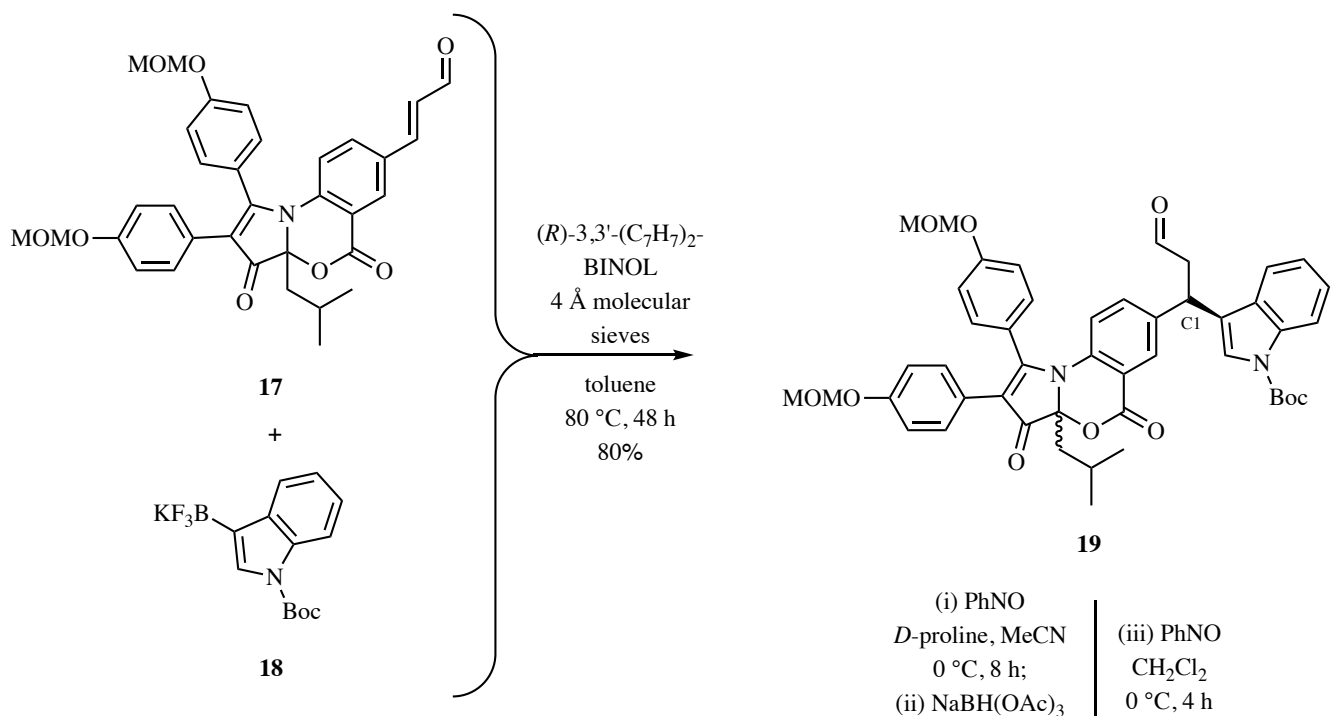

$$
\begin{array}{c|c}
\text { (i) } \mathrm{PhNO} & \\
D \text {-proline, } \mathrm{MeCN} & \text { (iii) } \mathrm{PhNO} \\
0{ }^{\circ} \mathrm{C}, 8 \mathrm{~h} ; & \mathrm{CH}_{2} \mathrm{Cl}_{2} \\
\text { (ii) } \mathrm{NaBH}(\mathrm{OAc})_{3} & 0{ }^{\circ} \mathrm{C}, 4 \mathrm{~h} \\
\mathrm{Cl}\left(\mathrm{CH}_{2}\right)_{2} \mathrm{Cl}, 22{ }^{\circ} \mathrm{C} & 36 \% \\
16 \mathrm{~h} &
\end{array}
$$
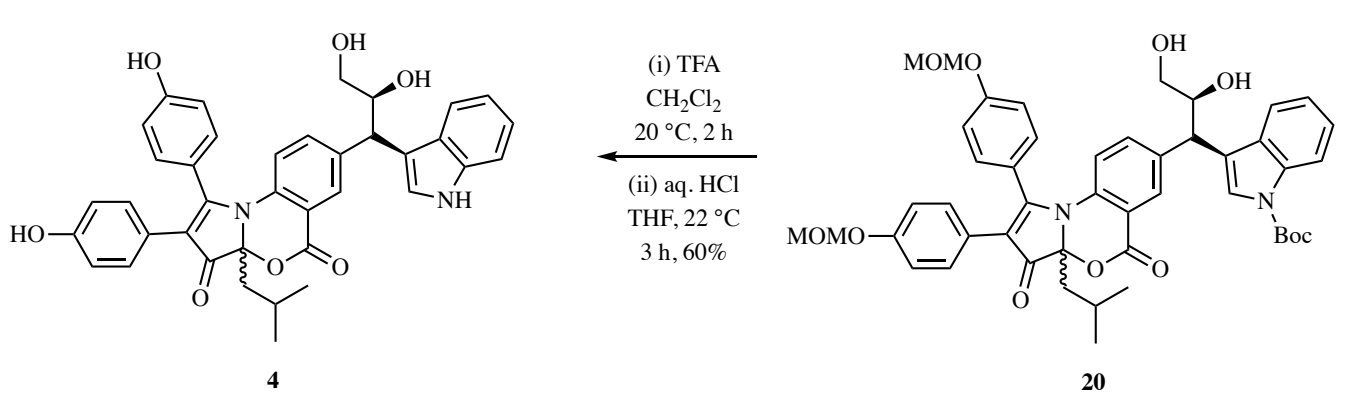

\section{CONCLUSION}

The total synthesis of discoipyrrole D reported here serves to confirm the basic structure (but not the absolute configuration) assigned to it by MacMillan and co-workers. This work also 
highlights the capacity of the protocols reported by May and his colleagues and to effect the organocatalyzed asymmetric conjugate addition of hetereoaryl trifluoroborates to cinnamaldehydes (in particular). In addition, the present study reveals more about the functionality that can be tolerated during the MoOPH-mediated formation of the abovementioned heterocyclic core and the various reaction conditions that can be used without adversely effecting this same and often rather fragile motif. 


\section{EXPERIMENTAL SECTION}

General Protocols. Unless otherwise specified, proton $\left({ }^{1} \mathrm{H}\right)$ and carbon $\left({ }^{13} \mathrm{C}\right)$ NMR spectra were recorded at $18{ }^{\circ} \mathrm{C}$ in base-filtered $\mathrm{CDCl}_{3}$ on a spectrometer operating at $400 \mathrm{MHz}$ for proton and $100 \mathrm{MHz}$ for carbon nuclei. ${ }^{1} \mathrm{H}$ NMR data are recorded as follows: chemical shift

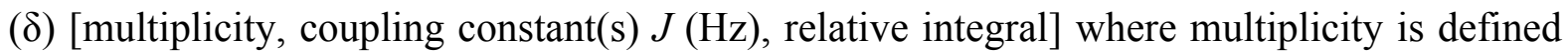
as $\mathrm{s}=$ singlet; $\mathrm{d}=$ doublet; $\mathrm{t}=$ triplet; $\mathrm{q}=$ quartet; $\mathrm{m}=$ multiplet or combinations of the above. In relevant cases, the signal due to residual $\mathrm{CHCl}_{3}$ appearing at $\delta_{\mathrm{H}} 7.26$ and the central resonance of the $\mathrm{CDCl}_{3}$ "triplet" appearing at $\delta_{\mathrm{C}} 77.0$ were used to reference ${ }^{1} \mathrm{H}$ and ${ }^{13} \mathrm{C}$ NMR spectra, respectively. Samples were analyzed by infrared spectroscopy $\left(v_{\max }\right)$ as thin films on $\mathrm{KBr}$ plates or as neat material resting on the sampling port. Low- and highresolution electron impact (EI) mass spectra were recorded on a double-focusing, triplesector machine. Low- and high-resolution ESI mass spectra were recorded on a triplequadrupole mass spectrometer operating in either positive or negative ion mode. Melting points are uncorrected. Analytical thin layer chromatography (TLC) was performed on aluminum-backed $0.2 \mathrm{~mm}$ thick silica gel $60 \mathrm{~F}_{254}$ plates. Eluted plates were visualized using a $254 \mathrm{~nm}$ UV lamp and/or by treatment with a suitable dip followed by heating. These dips included phosphomolybdic acid/ceric sulfate/sulfuric acid (conc.)/water (37.5 g : $7.5 \mathrm{~g}: 37.5$ $\mathrm{g}$ : $720 \mathrm{~mL}$ ), potassium permanganate/potassium carbonate $/ 5 \%$ sodium hydroxide aqueous solution/water (3 g : $20 \mathrm{~g}: 5 \mathrm{~mL}: 300 \mathrm{~mL}$ ), and $p$-anisaldehyde or vanillin/sulfuric acid (conc.)/ethanol (15 g : $2.5 \mathrm{~mL}: 250 \mathrm{~mL}$ ). Flash chromatographic separations were carried out following protocols defined by Still et al. ${ }^{13}$ with silica gel $60(40-63 \mu \mathrm{m})$ as the stationary phase and using the AR- or HPLC-grade solvents indicated. Starting materials, reagents, 
drying agents, and other inorganic salts were generally commercially available and were used as supplied. Tetrahydrofuran (THF), methanol and dichloromethane were dried using a solvent purification system that is based upon a technology originally described by Grubbs et al. ${ }^{14}$ Where necessary, reactions were performed under a nitrogen atmosphere.

Specific Chemical Transformations. Methyl 5-Bromo-2-(1H-pyrrol-1-yl)benzoate (7). A magnetically stirred and degassed mixture of pyrrole (2) (1.39 g, $20.8 \mathrm{mmol})$, commercially available compound 4 (6.45 g, $18.9 \mathrm{mmol})$, CuI (359 mg, $1.9 \mathrm{mmol}), 1,10-$ phenanthroline $(680 \mathrm{mg}, 3.8 \mathrm{mmol})$ and $\mathrm{Cs}_{2} \mathrm{CO}_{3}(9.30 \mathrm{~g}, 28.4 \mathrm{mmol})$ in anhydrous toluene $(40 \mathrm{~mL})$ was heated at $100{ }^{\circ} \mathrm{C}$ under a nitrogen atmosphere for $48 \mathrm{~h}$. The cooled reaction mixture was then passed through a pad of TLC-grade silica and the filtrate concentrated under reduced pressure. The residue so formed was subjected to flash chromatography (silica, 30:1 v/v hexane/ethyl acetate elution) to afford, after concentration of the appropriate fractions $\left(R_{f}=0.5\right.$ in $8: 1 \mathrm{v} / \mathrm{v}$ hexane/ethyl acetate), compound $7(5.21 \mathrm{~g}, 99 \%)$ as a clear, colorless syrup. ${ }^{1} \mathrm{H}$ NMR $\left(400 \mathrm{MHz}, \mathrm{CDCl}_{3}\right) \delta 7.85(\mathrm{~d}, J=2.4 \mathrm{~Hz}, 1 \mathrm{H}), 7.59(\mathrm{dd}, J=8.5$ and $2.4 \mathrm{~Hz}, 2 \mathrm{H}), 7.18(\mathrm{~m}, 1 \mathrm{H}), 6.71(\mathrm{~m}, 2 \mathrm{H}), 6.25(\mathrm{~m}, 2 \mathrm{H}), 3.65$ (s, 3H); ${ }^{13} \mathrm{C} \mathrm{NMR}(100 \mathrm{MHz}$, $\left.\mathrm{CDCl}_{3}\right) \delta 166.1,139.4,135.3,133.5,129.4,128.3,122.0,120.5,110.2,52.8 ; \mathrm{IR} v_{\max } 2950$ $1729,1594,1563,1498,1435,1400,1329,1288,1267,1238,1123,1094,1015,966,922$, 826, $727 \mathrm{~cm}^{-1}$; MS (ESI, +ve): $m / z 282$ and $280\left[(\mathrm{M}+\mathrm{H})^{+}\right.$, both 50\%], 250 and $248(96$ and 100); HRMS (ESI, +ve) Found: $(\mathrm{M}+\mathrm{H})^{+}$, 279.9972. $\mathrm{C}_{12} \mathrm{H}_{11}{ }^{79} \mathrm{BrNO}_{2}$ requires $(\mathrm{M}+\mathrm{H})^{+}$, 279.9973.

Methyl 5-Bromo-2-(2-formyl-1H-pyrrol-1-yl)benzoate (8). Anhydrous DMF/THF (90 mL of a $4: 5 \mathrm{v} / \mathrm{v}$ mixture) maintained with magnetic stirring at $0{ }^{\circ} \mathrm{C}$ under a nitrogen atmosphere was treated with $\mathrm{POCl}_{3}(5.50 \mathrm{~mL}, 59.5 \mathrm{mmol})$ and the resulting orange reaction 
mixture was stirred at $0{ }^{\circ} \mathrm{C}$ for $0.75 \mathrm{~h}$ before being treated, dropwise, with a solution of compound 7 (6.47 g, $23.2 \mathrm{mmol})$ in anhydrous THF $(40 \mathrm{~mL})$. The mixture so-formed was warmed to $22{ }^{\circ} \mathrm{C}$ then stirred at this temperature for $3 \mathrm{~h}$ before being quenched with ice (100 g). The ensuing mixture was neutralized using $\mathrm{NaHCO}_{3}$ (saturated aqueous solution) then extracted with diethyl ether $(3 \times 150 \mathrm{~mL})$. The combined organic phases were washed with brine $(1 \times 300 \mathrm{~mL})$ before being dried $\left(\mathrm{Na}_{2} \mathrm{SO}_{4}\right)$, filtered and then concentrated under reduced pressure. The residue thus obtained was subjected to flash chromatography (silica, 12:1 $\mathrm{v} / \mathrm{v}$ hexane/ethyl acetate elution) to afford, after concentration of the appropriate fractions $\left(R_{f}=0.3\right.$ in $4: 1 \mathrm{v} / \mathrm{v}$ hexane/ethyl acetate), compound $8(5.89 \mathrm{~g}, 83 \%)$ as a clear, colorless syrup. ${ }^{1} \mathrm{H}$ NMR $\left(400 \mathrm{MHz}, \mathrm{CDCl}_{3}\right) \delta 9.48(\mathrm{~s}, 1 \mathrm{H}), 8.16(\mathrm{~d}, J=2.4 \mathrm{~Hz}, 1 \mathrm{H}), 7.71$ (dd, $J=8.3$ and $2.4 \mathrm{~Hz}, 1 \mathrm{H}), 7.19(\mathrm{~d}, J=8.3 \mathrm{~Hz}, 1 \mathrm{H}), 7.09(\mathrm{dd}, J=4.0$ and $1.7 \mathrm{~Hz}, 1 \mathrm{H}), 6.94$ (m, 1H), 6.43 (broadened s, 1H), $3.68(\mathrm{~s}, 3 \mathrm{H}) ;{ }^{13} \mathrm{C} \mathrm{NMR}\left(100 \mathrm{MHz}, \mathrm{CDCl}_{3}\right) \delta$ 178.7, 164.1, $138.5,135.6,134.0,133.2,131.6,130.4,129.9,123.8,122.5,110.9,52.6$; IR $v_{\max } 3100$ $2843,1727,1646,1489,1415,1361,1284,1246,1088,1075,1039,836,761,745 \mathrm{~cm}^{-1}$; MS (ESI, +ve): $\mathrm{m} / z 332$ and 330 [(M+Na) ${ }^{+}, 95$ and 100\%], 310 and 308 (both 6); HRMS (ESI, +ve) Found: $(\mathrm{M}+\mathrm{H})^{+}$, 307.9927. $\mathrm{C}_{13} \mathrm{H}_{11}{ }^{79} \mathrm{BrNO}_{3}$ requires $(\mathrm{M}+\mathrm{H})^{+}, 307.9922$.

\section{Methyl 5-Bromo-2-(5-formyl-2,3-diiodo-1 $H$-pyrrol-1-yl)benzoate (9). A} magnetically stirred mixture of compound $8(5.89 \mathrm{~g}, 19.2 \mathrm{mmol})$ and $\mathrm{CF}_{3} \mathrm{COOAg}_{\text {in dry THF }}$ $(80 \mathrm{~mL})$ maintained at $0{ }^{\circ} \mathrm{C}$ under a nitrogen atmosphere was treated with molecular iodine $(9.99 \mathrm{~g}, 39.3 \mathrm{mmol})$ and the resulting deep-red reaction mixture was warmed to $22{ }^{\circ} \mathrm{C}$ over $16 \mathrm{~h}$ while being protected from light. After this time the reaction mixture was filtered through a pad of TLC-grade silica and the filtrate concentrated under reduced pressure. The residue thus obtained was subjected to flash chromatography (silica, 9:1 v/v hexane/THF 
elution) to afford, after concentration of the appropriate fractions $\left(R_{f}=0.5\right.$ in $4: 1 \mathrm{v} / \mathrm{v}$ hexane/ethyl acetate), compound 9 (8.21 g, 77\%) as an amorphous solid. ${ }^{1} \mathrm{H}$ NMR (400 $\left.\mathrm{MHz}, \mathrm{CDCl}_{3}\right) \delta 9.13(\mathrm{~s}, 1 \mathrm{H}), 8.27(\mathrm{~d}, J=2.3 \mathrm{~Hz}, 1 \mathrm{H}), 7.79(\mathrm{dd}, J=8.4$ and $2.3 \mathrm{~Hz}, 1 \mathrm{H}), 7.21$ (s, $1 \mathrm{H}), 7.09(\mathrm{~d}, J=8.4 \mathrm{~Hz}, 1 \mathrm{H}), 3.70(\mathrm{~s}, 3 \mathrm{H}) ;{ }^{13} \mathrm{C} \mathrm{NMR}\left(100 \mathrm{MHz}, \mathrm{CDCl}_{3}\right) \delta 176.5,163.2$ 139.4, 138.5, 136.3, 134.7, 131.8, 130.0, 129.7, 124.0, 100.2, 78.1, 52.9; IR $v_{\max } 3446,3110$, $2950,1730,1670,1488,1435,1380,1351,1287,1254,1096,835 \mathrm{~cm}^{-1}$; MS (ESI, +ve) $\mathrm{m} / z$ 584 and $582\left[(\mathrm{M}+\mathrm{Na})^{+}, 100\right.$ and 97\%], 562 and $560\left[(\mathrm{M}+\mathrm{H})^{+}\right.$, both 33]; HRMS (ESI, +ve) Found: $(\mathrm{M}+\mathrm{H})^{+}$, 559.7855. $\mathrm{C}_{13} \mathrm{H}_{9}{ }^{79} \mathrm{Br}^{127} \mathrm{I}_{2} \mathrm{NO}_{3}$ requires $(\mathrm{M}+\mathrm{H})^{+}, 559.7855$.

Methyl 5-Bromo-2-(5-formyl-2,3-bis(4-hydroxyphenyl)-1 H-pyrrol-1y1)benzoate (11). A magnetically stirred and degassed mixture of compound 9 (1.62 g, 2.91 mmol), commercially available boronic acid 10 (910 mg, $6.40 \mathrm{mmol}), \operatorname{Pd}\left(\mathrm{PPh}_{3}\right)_{2} \mathrm{Cl}_{2}(163 \mathrm{mg}$, $0.23 \mathrm{mmol})$ and $\mathrm{Na}_{2} \mathrm{CO}_{3}(1.23 \mathrm{~g}, 11.64 \mathrm{mmol})$ in acetonitrile/water $(75 \mathrm{~mL}$ of a $3: 2 \mathrm{v} / \mathrm{v}$ mixture) was heated at $60{ }^{\circ} \mathrm{C}$ for $48 \mathrm{~h}$ while being maintained under a nitrogen atmosphere throughout this period. The cooled reaction mixture was passed through a pad of TLC-grade silica and the filtrate concentrated under reduced pressure. The residue thus obtained was subject to flash chromatography (silica, 2:1 v/v hexane/ethyl acetate elution) to afford, after concentration of the appropriate fractions $\left(R_{f}=0.3\right.$ in $1: 1 \mathrm{v} / \mathrm{v}$ hexane/ethyl acetate $)$, compound $11(1.20 \mathrm{~g}, 84 \%)$ as a pale-yellow foam. ${ }^{1} \mathrm{H}$ NMR $\left(400 \mathrm{MHz}, \mathrm{CD}_{3} \mathrm{OD}\right) \delta 9.38(\mathrm{~s}$, $1 \mathrm{H}), 8.00(\mathrm{~d}, J=2.3 \mathrm{~Hz}, 1 \mathrm{H}), 7.69(\mathrm{dd}, J=8.3$ and $2.3 \mathrm{~Hz}, 1 \mathrm{H}), 7.36(\mathrm{~s}, 1 \mathrm{H}), 7.21(\mathrm{~d}, J=8.3$ $\mathrm{Hz}, 1 \mathrm{H}), 7.07(\mathrm{~d}, J=8.6 \mathrm{~Hz}, 2 \mathrm{H}), 6.86(\mathrm{~d}, J=8.6 \mathrm{~Hz}, 2 \mathrm{H}), 6.67(\mathrm{~d}, J=8.6 \mathrm{~Hz}, 2 \mathrm{H}), 6.61(\mathrm{~d}$, $J=8.6 \mathrm{~Hz}, 2 \mathrm{H}), 3.69(\mathrm{~s}, 3 \mathrm{H})$ (signals due to protons of phenolic hydroxyl groups not observed); ${ }^{13} \mathrm{C}$ NMR (100 MHz, $\left.\mathrm{CD}_{3} \mathrm{OD}\right) \delta 180.1,165.6,159.0,157.2,142.0,138.7,136.3$, $134.3,133.9,133.6,133.3,132.5,130.2,127.1,127.0,124.5,123.0,122.3,116.2,116.1$, 
53.0; IR $v_{\max } 3315,2954,2873,1732,1712,1636,1612,1457,1434,1419,1258,1230$, 1159, 1100, 830, $736 \mathrm{~cm}^{-1}$; MS (ESI, +ve) $\mathrm{m} / z 516$ and $514\left[(\mathrm{M}+\mathrm{Na})^{+}, 93\right.$ and 100\%], 494 and $492\left[(\mathrm{M}+\mathrm{H})^{+}, 20\right.$ and 19]; HRMS (ESI, +ve) Found: $(\mathrm{M}+\mathrm{Na})^{+}, \quad 514.0265$. $\mathrm{C}_{25} \mathrm{H}_{18}{ }^{79} \mathrm{BrNNaO}_{5}$ requires $(\mathrm{M}+\mathrm{Na})^{+}, 514.0266$.

Methyl 5-Bromo-2-(5-formyl-2,3-bis(4-(methoxymethoxy)phenyl)-1 $\mathrm{H}$-pyrrol1-yl)benz- oate (12). A magnetically stirred solution of compound 11 (1.26 g, $2.57 \mathrm{mmol})$ and $N, N$-di-isopropylethylamine $(3.32 \mathrm{~g}, 25.7 \mathrm{mmol})$ in dry dichloromethane $(25 \mathrm{~mL})$ maintained at $0{ }^{\circ} \mathrm{C}$ under a nitrogen atmosphere was treated with freshly prepared $\mathrm{MOMCl}$ (12 $\mathrm{mL}$ of an $2.14 \mathrm{M}$ solution in dry dichloromethane, $25.7 \mathrm{mmol}$ ). The resulting lightyellow reaction mixture was warmed to $22{ }^{\circ} \mathrm{C}$ over $16 \mathrm{~h}$ then treated, successively, with $\mathrm{NH}_{4} \mathrm{Cl}(50 \mathrm{~mL}$ of a saturated aqueous solution) and water $(100 \mathrm{~mL})$ before being extracted with ethyl acetate $(3 \times 100 \mathrm{~mL})$. The combined organic phases were washed with brine $(1 \times$ $150 \mathrm{~mL})$ then dried $\left(\mathrm{Na}_{2} \mathrm{SO}_{4}\right)$, filtered and concentrated under reduced pressure. The residue thus obtained was subjected to flash chromatography (silica, 4:1 v/v hexane/ethyl acetate elution) and concentration of the appropriate fractions $\left(R_{f}=0.6\right.$ in $1: 1 \mathrm{v} / \mathrm{v}$ hexane/ethyl acetate) gave compound $12(1.51 \mathrm{~g}, 99 \%)$ as a pale-yellow foam. ${ }^{1} \mathrm{H}$ NMR (400 MHz, $\left.\mathrm{CDCl}_{3}\right) \delta 9.47(\mathrm{~s}, 1 \mathrm{H}), 8.04(\mathrm{~d}, J=2.3 \mathrm{~Hz}, 1 \mathrm{H}), 7.56(\mathrm{dd}, J=8.4$ and $2.3 \mathrm{~Hz}, 1 \mathrm{H}), 7.24(\mathrm{~s}$, 1H), $7.13(\mathrm{~d}, J=8.8 \mathrm{~Hz}, 2 \mathrm{H}), 7.07(\mathrm{~d}, J=8.4 \mathrm{~Hz}, 1 \mathrm{H}), 6.95-6.89$ (complex m, 4H), 6.81 (d, $J=8.8 \mathrm{~Hz}, 2 \mathrm{H}), 5.14(\mathrm{~s}, 2 \mathrm{H}), 5.11(\mathrm{~s}, 2 \mathrm{H}), 3.70(\mathrm{~s}, 3 \mathrm{H}), 3.47$ (s, 3H), $3.45(\mathrm{~s}, 3 \mathrm{H}) ;{ }^{13} \mathrm{C} \mathrm{NMR}$ $\left(100 \mathrm{MHz}, \mathrm{CDCl}_{3}\right) \delta 178.5,164.0,157.4,156.0,139.5,137.2,135.3,133.8,132.5,132.2$ $132.0,130.9,129.2,128.2,125.2,123.4,123.0,122.4,116.2,116.0,94.5,94.4,56.3,56.1$, 52.6; IR $v_{\max } 2953,2902,2827,1732,1662,1461,1286,1235,1151,1077,994,836 \mathrm{~cm}^{-1}$; MS (ESI, +ve) $m / z 604$ and $602\left[(\mathrm{M}+\mathrm{Na})^{+}, 100\right.$ and 97\%], 582 and $580\left[(\mathrm{M}+\mathrm{H})^{+}, 33\right.$ and 28]; 
HRMS (ESI, +ve) Found: $(\mathrm{M}+\mathrm{Na})^{+}$, 602.0794. $\mathrm{C}_{29} \mathrm{H}_{26}{ }^{79} \mathrm{BrNNaO}_{7}$ requires $(\mathrm{M}+\mathrm{Na})^{+}$, 602.0790 .

\section{Methyl 2-(2,3-bis(4-(Methoxymethoxy)phenyl)-5-(2-methylprop-1-en-1-yl)-} $1 H$-pyrrol-1-yl)-5-bromobenzoate (13). A magnetically stirred suspension of $i-\mathrm{PrPPh}_{3} \mathrm{I}$ $(1.44 \mathrm{~g}, 3.16 \mathrm{mmol})$ in dry THF $(20 \mathrm{~mL})$ maintained at $-78^{\circ} \mathrm{C}$ under a nitrogen atmosphere was treated with $n$-BuLi $(1.82 \mathrm{~mL}$ of a $1.6 \mathrm{M}$ solution in hexane, $2.91 \mathrm{mmol})$, and the ensuing red suspension stirred at $-78^{\circ} \mathrm{C}$ for $0.5 \mathrm{~h}$ before being added, over $0.17 \mathrm{~h}$, to a magnetically solution of compound $\mathbf{1 2}(1.41 \mathrm{~g}, 2.43 \mathrm{mmol})$ in dry THF $(40 \mathrm{~mL})$ maintained at $-78{ }^{\circ} \mathrm{C}$. The reaction mixture thus formed was transferred to an ice-water bath and maintained at $c a .0{ }^{\circ} \mathrm{C}$ for $1 \mathrm{~h}$ then treated, successively, with $\mathrm{NH}_{4} \mathrm{Cl}(10 \mathrm{~mL}$ of a saturated aqueous solution) and water $(40 \mathrm{~mL})$ before being extracted with ethyl acetate $(3 \times 50 \mathrm{~mL})$. The combined organic phases were washed with brine $(1 \times 100 \mathrm{~mL})$ then dried $\left(\mathrm{Na}_{2} \mathrm{SO}_{4}\right)$, filtered and concentrated under reduced pressure. The residue thus obtained was subjected to flash chromatography (silica, 15:1 $\mathrm{v} / \mathrm{v}$ hexane/ethyl acetate elution) to afford, after concentration of the appropriate fractions $\left(R_{f}=0.6\right.$ in $4: 1 \mathrm{v} / \mathrm{v}$ hexane/ethyl acetate), compound $13(1.16 \mathrm{~g}, 79 \%)$ as a pale-yellow foam. ${ }^{1} \mathrm{H}$ NMR (400 MHz, $\left.\mathrm{CDCl}_{3}\right) \delta 7.93(\mathrm{~d}, J$ $=2.4 \mathrm{~Hz}, 1 \mathrm{H}), 7.55(\mathrm{dd}, J=8.4$ and $2.4 \mathrm{~Hz}, 1 \mathrm{H}), 7.17(\mathrm{~d}, J=8.8 \mathrm{~Hz}, 2 \mathrm{H}), 7.04(\mathrm{~d}, J=8.4$ Hz, 1H), $6.90(\mathrm{~m}, 4 \mathrm{H}), 6.78(\mathrm{~d}, J=8.8 \mathrm{~Hz}, 2 \mathrm{H}), 6.45(\mathrm{~s}, 1 \mathrm{H}), 5.49(\mathrm{~s}, 1 \mathrm{H}), 5.14(\mathrm{~s}, 2 \mathrm{H}), 5.11$ (s, 2H), $3.64(\mathrm{~s}, 3 \mathrm{H}), 3.48(\mathrm{~s}, 3 \mathrm{H}), 3.46(\mathrm{~s}, 3 \mathrm{H}), 1.97(\mathrm{~s}, 3 \mathrm{H}), 1.77$ (s, 3H); ${ }^{13} \mathrm{C}$ NMR $(100$ $\left.\mathrm{MHz}, \mathrm{CDCl}_{3}\right) \delta 164.9,156.4,155.3,137.6,135.3,135.0,133.6,132.8,132.3,132.2(4)$, 132.2(0), 130.4, 129.5, 129.1, 126.0, 122.8, 121.4, 116.1, 115.9, 114.5, 109.4, 94.7, 94.6, 56.2, 56.0, 52.6, 27.0, 20.3; IR $v_{\max }$ 2951, 2900, 1733, 1515, 1486, 1284, 1232, 1151, 1077, 999, 834, $731 \mathrm{~cm}^{-1}$; MS (ESI, +ve) $\mathrm{m} / z 630$ and $628\left[(\mathrm{M}+\mathrm{Na})^{+}, 100\right.$ and 90\%], 608 and 606 
$\left[(\mathrm{M}+\mathrm{H})^{+}, 38\right.$ and 40]; HRMS (ESI, +ve) Found: $(\mathrm{M}+\mathrm{H})^{+}, 606.1494 . \mathrm{C}_{32} \mathrm{H}_{33}{ }^{79} \mathrm{BrNO}_{6}$ requires $(\mathrm{M}+\mathrm{H})^{+}, 606.1491$.

Methyl 5-bromo-2-(5-isobutyl-2,3-bis(4-(methoxymethoxy)phenyl)-1H-pyrrol-1-yl)benzoate (14). A magnetically stirred mixture of compound 13 (4.72 g, $7.80 \mathrm{mmol})$ in dry THF $(100 \mathrm{~mL})$ was treated with $\mathrm{PtO}_{2} \cdot \mathrm{H}_{2} \mathrm{O}(573 \mathrm{mg}, 2.34 \mathrm{mmol})$ and the ensuing black suspension stirred at $22{ }^{\circ} \mathrm{C}$ under a balloon of hydrogen for $48 \mathrm{~h}$ then filtered through a pad of TLCgrade silica. The filtrate was concentrated under reduced pressure and the residue subjected to flash chromatography (silica, $9: 1 \mathrm{v} / \mathrm{v}$ hexane/ethyl acetate elution) to afford, after concentration of the appropriate fractions $\left(R_{f}=0.6\right.$ in $4: 1 \mathrm{v} / \mathrm{v}$ hexane/ethyl acetate), compound $14(4.70 \mathrm{~g}, 99 \%)$ as a pale-yellow foam. ${ }^{1} \mathrm{H}$ NMR (400 MHz, $\left.\mathrm{CDCl}_{3}\right) \delta 7.92(\mathrm{~d}, J$ $=2.4 \mathrm{~Hz}, 1 \mathrm{H}), 7.60(\mathrm{dd}, J=8.4$ and $2.4 \mathrm{~Hz}, 1 \mathrm{H}), 7.18-7.11$ (complex m, 3H), 6.91-6.85 (complex m, 4H), $6.76(\mathrm{~d}, J=8.7 \mathrm{~Hz}, 2 \mathrm{H}), 6.24(\mathrm{~s}, 1 \mathrm{H}), 5.13(\mathrm{~s}, 2 \mathrm{H}), 5.10(\mathrm{~s}, 2 \mathrm{H}), 3.64(\mathrm{~s}$, 3H), $3.47(\mathrm{~s}, 3 \mathrm{H}), 3.46(\mathrm{~s}, 3 \mathrm{H}), 2.25(\mathrm{dd}, J=15.2$ and $7.0 \mathrm{~Hz}, 1 \mathrm{H}), 2.16(\mathrm{dd}, J=15.2$ and 7.2 $\mathrm{Hz}, 1 \mathrm{H}), 1.70(\mathrm{~m}, 1 \mathrm{H}), 0.88(\mathrm{~m}, 6 \mathrm{H}) ;{ }^{13} \mathrm{C} \mathrm{NMR}\left(100 \mathrm{MHz}, \mathrm{CDCl}_{3}\right) \delta 164.8,156.3,155.1$, $138.0,135.2,133.9,133.8,132.8,132.3,132.2,130.7,129.6,128.9,126.4,122.0,121.6$, $116.1,115.8,107.8,94.7,94.6,56.3,56.1,52.6,36.3,27.8,22.7(8), 22.7(5)$; IR $v_{\max } 2952$, 2899, 1737, 1515, 1486, 1283, 1233, 1151, 1078, 999, $836 \mathrm{~cm}^{-1}$; MS (ESI, +ve) $\mathrm{m} / z 610$ and $608\left[(\mathrm{M}+\mathrm{H})^{+}, 100\right.$ and 92\%], 632 and $630\left[(\mathrm{M}+\mathrm{Na})^{+}, 90\right.$ and 88]; HRMS (ESI, +ve) Found: $(\mathrm{M}+\mathrm{H})^{+}, 608.1647 . \mathrm{C}_{32} \mathrm{H}_{35}{ }^{79} \mathrm{BrNO}_{6}$ requires $(\mathrm{M}+\mathrm{H})^{+}, 608.1648$.

Methyl (E)-5-(3,3-Diethoxyprop-1-en-1-yl)-2-(5-isobutyl-2,3-bis(4(methoxymethoxy)-phenyl)-1H-pyrrol-1-yl)benzoate (15). A magnetically stirred mixture of compound 14 (647 mg, $1.06 \mathrm{mmol})$, acrolein diethyl acetal (1.38 g, $10.6 \mathrm{mmol})$, tetra- $n$-butylammonium acetate $(640 \mathrm{mg}, 2.12 \mathrm{mmol}), \mathrm{K}_{2} \mathrm{CO}_{3}(220 \mathrm{mg}, 1.59 \mathrm{mmol}), \mathrm{KCl}(80$ 
$\mathrm{mg}, 1.06 \mathrm{mmol})$ and $\mathrm{Pd}(\mathrm{OAc})_{2}(120 \mathrm{mg}, 0.53 \mathrm{mmol})$ in anhydrous DMF $(10 \mathrm{~mL})$ was heated at $100{ }^{\circ} \mathrm{C}$ in a sealed tube for $48 \mathrm{~h}$. The cooled reaction mixture was filtered through a pad of TLC-grade silica and the filtrate concentrated under reduced pressure. The residue thus obtained was subjected to flash chromatography (silica, 9:1 v/v hexane/ethyl acetate elution) to afford, after concentration of the appropriate fractions $\left(R_{f}=0.5\right.$ in $4: 1 \mathrm{v} / \mathrm{v}$ hexane/ethyl acetate), compound $15(453 \mathrm{mg}, 65 \%)$ as a clear, yellow oil. ${ }^{1} \mathrm{H}$ NMR [400 MHz, $\left(\mathrm{CD}_{3}\right)_{2} \mathrm{CO}$ ] $\delta 7.86(\mathrm{~s}, 1 \mathrm{H}), 7.73(\mathrm{~d}, J=8.1 \mathrm{~Hz}, 1 \mathrm{H}), 7.37(\mathrm{~d}, J=8.1 \mathrm{~Hz}, 1 \mathrm{H}), 7.18(\mathrm{~d}, J=8.6 \mathrm{~Hz}, 2 \mathrm{H})$, $7.00(\mathrm{~d}, J=8.5 \mathrm{~Hz}, 2 \mathrm{H}), 6.88(\mathrm{~d}, J=8.6 \mathrm{~Hz}, 2 \mathrm{H}), 6.80(\mathrm{~m}, 3 \mathrm{H}), 6.36(\mathrm{dd}, J=16.2$ and 4.9 $\mathrm{Hz}, 1 \mathrm{H}), 6.27(\mathrm{~s}, 1 \mathrm{H}), 5.15(\mathrm{~s}, 2 \mathrm{H}), 5.10(\mathrm{~m}, 3 \mathrm{H}), 3.73-3.64$ (complex m, 5H), $3.55(\mathrm{~m}, 2 \mathrm{H})$ $3.43(\mathrm{~s}, 3 \mathrm{H}), 3.39(\mathrm{~s}, 3 \mathrm{H}), 2.33(\mathrm{dd}, J=15.0$ and $7.1 \mathrm{~Hz}, 1 \mathrm{H}), 2.23(\mathrm{dd}, J=15.0$ and $7.2 \mathrm{~Hz}$, 1H), $1.70(\mathrm{~m}, 1 \mathrm{H}), 1.20(\mathrm{~m}, 6 \mathrm{H}), 0.89(\mathrm{~m}, 6 \mathrm{H}) ;{ }^{13} \mathrm{C}$ NMR [100 MHz, $\left.\left(\mathrm{CD}_{3}\right)_{2} \mathrm{CO}\right] \delta 166.5$, 157.4, 156.2, 138.9, 137.4, 134.5, 133.3, 132.8, 132.2, 131.9, 131.4, 130.7, 129.7, 129.5, $127.7,122.6,116.9,116.5,108.5,101.9,95.4,95.3,61.7,56.3,56.1,52.7,37.2,28.6,23.1$, 15.9, 15.8; IR $v_{\max } 2853,2898,1719,1515,1302,1232,1198,1150,1077,997,921,837,788$ $\mathrm{cm}^{-1}$; MS (ESI, +ve): $\mathrm{m} / z 680\left[(\mathrm{M}+\mathrm{Na})^{+}, 15 \%\right], 658\left[(\mathrm{M}+\mathrm{H})^{+}, 100\right]$; HRMS $(\mathrm{ESI},+\mathrm{ve})$ Found: $(\mathrm{M}+\mathrm{H})^{+}, 658.3389 . \mathrm{C}_{39} \mathrm{H}_{48} \mathrm{NO}_{8}$ requires $(\mathrm{M}+\mathrm{H})^{+}, 658.3380$.

(E)-2-(5-Isobutyl-2,3-bis(4-(methoxymethoxy)phenyl)-1 H-pyrrol-1-yl)-5-(3oxoprop-1-en-1-yl)benzoic acid (16). A magnetically stirred solution of compound 15 (453 mg, $0.69 \mathrm{mmol})$ in THF/water/ethanol $(20 \mathrm{~mL}$ of a 1:1:2 v/v/v mixture) was treated with $\mathrm{KOH}(386 \mathrm{mg}, 6.9 \mathrm{mmol})$ and the ensuing mixture stirred at $22{ }^{\circ} \mathrm{C}$ for $24 \mathrm{~h}$ then acidified, using $\mathrm{HCl}$ (2 $\mathrm{M}$ aqueous solution), to $\mathrm{pH} 2$. The mixture thus obtained was diluted with brine $(50 \mathrm{~mL})$ and then extracted with ethyl acetate $(3 \times 50 \mathrm{~mL})$. The combined organic phases were washed with brine $(3 \times 100 \mathrm{~mL})$ before being dried $\left(\mathrm{Na}_{2} \mathrm{SO}_{4}\right)$, filtered, and then 
concentrated under reduced pressure. The residue thus obtained was subjected to flash chromatography (silica, 3:1 v/v hexane/acetone elution) to afford, after concentration of the appropriate fractions $\left(R_{f}=0.6\right.$ in $1: 1 \mathrm{v} / \mathrm{v}$ hexane/acetone), compound $16(353 \mathrm{mg}, 90 \%)$ as a light-yellow oil. ${ }^{1} \mathrm{H}$ NMR $\left(400 \mathrm{MHz}, \mathrm{CDCl}_{3}\right) \delta 9.71(\mathrm{~d}, J=7.5 \mathrm{~Hz}, 1 \mathrm{H}), 8.06(\mathrm{~d}, J=1.6 \mathrm{~Hz}$, 1H), $7.71(\mathrm{dd}, J=8.3$ and $2.1 \mathrm{~Hz}, 1 \mathrm{H}), 7.45(\mathrm{~d}, J=16.0 \mathrm{~Hz}, 1 \mathrm{H}), 7.31(\mathrm{~d}, J=8.2 \mathrm{~Hz}, 1 \mathrm{H})$, $7.12(\mathrm{~d}, J=8.6 \mathrm{~Hz}, 2 \mathrm{H}), 6.91-6.84$ (complex m, 4H), 6.75 (dd, $J=16.0$ and $7.6 \mathrm{~Hz}, 1 \mathrm{H})$, $6.69(\mathrm{~d}, J=8.4 \mathrm{~Hz}, 2 \mathrm{H}), 6.24(\mathrm{~s}, 1 \mathrm{H}), 5.12(\mathrm{~s}, 2 \mathrm{H}), 5.04(\mathrm{~s}, 2 \mathrm{H}), 3.46(\mathrm{~s}, 3 \mathrm{H}), 3.41(\mathrm{~s}, 3 \mathrm{H})$ $2.29(\mathrm{dd}, J=15.2$ and $7.0 \mathrm{~Hz}, 1 \mathrm{H}), 2.19(\mathrm{dd}, J=15.2$ and $7.2 \mathrm{~Hz}, 1 \mathrm{H}), 1.66(\mathrm{~m}, 1 \mathrm{H}), 0.85$ $(\mathrm{m}, 6 \mathrm{H})$ (signal due to carboxylic acid group proton not observed); ${ }^{13} \mathrm{C} \mathrm{NMR}(100 \mathrm{MHz}$, $\left.\mathrm{CDCl}_{3}\right) \delta 193.4,169.1,156.3,155.1,150.1,141.7,133.6(3), 133.5(9), 132.5,131.9,131.8$ $130.5,130.2,129.6,129.0,126.3,122.3,116.1,115.9,108.4,94.7,94.6,56.2,56.1,36.4$ 27.9, 22.7(2), 22.6(8); IR $v_{\max } 2954,1680,1515,1232,1198,1150,1121,1078,999,920$, $837,731 \mathrm{~cm}^{-1}$; MS (ESI, +ve) $m / z 570\left[(\mathrm{M}+\mathrm{H})^{+}, 100 \%\right]$; HRMS (ESI, +ve) Found: $(\mathrm{M}+\mathrm{H})^{+}$, 570.2496. $\mathrm{C}_{34} \mathrm{H}_{36} \mathrm{NO}_{7}$ requires $(\mathrm{M}+\mathrm{H})^{+}, 570.2492$.

(E)-3-(3a-Isobutyl-1,2-bis(4-(methoxymethoxy)phenyl)-3,5-dioxo-3,3adihydro-5 $H$-ben-zo[ $d]$ pyrrole $[2,1-b][1,3]$ oxazin-7-yl)acrylaldehyde $\quad(17) . \quad$ A magnetically stirred solution of compound $16(540 \mathrm{mg}, 0.95 \mathrm{mmol})$ in dry methanol $(25 \mathrm{~mL})$ maintained under a nitrogen atmosphere at $22{ }^{\circ} \mathrm{C}$ was treated with $\mathrm{MoOPH}(825 \mathrm{mg}, 1.9$ mmol). The ensuing yellow-colored reaction mixture was stirred, while being protected from light, at $22{ }^{\circ} \mathrm{C}$ for $16 \mathrm{~h}$ then filtered through a pad of TLC-grade silica. The filtrate was concentrated under reduced pressure and the residue thus obtained subjected to flash chromatography (silica, 2:1 v/v hexane/ethyl acetate elution) to afford, after concentration of the appropriate fractions $\left(R_{f}=0.3\right.$ in $2: 1 \mathrm{v} / \mathrm{v}$ hexane/ethyl acetate), compound 17 (277 $\mathrm{mg}$, 
$50 \%)$ as a light-yellow oil. ${ }^{1} \mathrm{H}$ NMR $\left(400 \mathrm{MHz}, \mathrm{CDCl}_{3}\right) \delta 9.69(\mathrm{~d}, J=7.5 \mathrm{~Hz}, 1 \mathrm{H}), 8.24(\mathrm{~d}, J$ $=2.1 \mathrm{~Hz}, 1 \mathrm{H}), 7.50(\mathrm{dd}, J=8.8$ and $2.2 \mathrm{~Hz}, 1 \mathrm{H}), 7.40(\mathrm{~d}, J=16.0 \mathrm{~Hz}, 1 \mathrm{H}), 7.19-7.06$ (complex m, 6H), $6.88(\mathrm{~d}, J=8.8 \mathrm{~Hz}, 2 \mathrm{H}), 6.66(\mathrm{dd}, J=16.0$ and $7.5 \mathrm{~Hz}, 1 \mathrm{H}), 6.34(\mathrm{~d}, J=$ 8.6 Hz, 1H), 5.26-5.21 (complex m, 2H), 5.14-5.09 (complex m, 2H), 3.53 (s, 3H), 3.44 (s, 3H), $2.35(\mathrm{dd}, J=14.1$ and $5.7 \mathrm{~Hz}, 1 \mathrm{H}), 1.97(\mathrm{dd}, J=14.1$ and $7.0 \mathrm{~Hz}, 1 \mathrm{H}), 1.83-1.71$ (complex m, 1H), $0.94(\mathrm{~d}, J=6.7 \mathrm{~Hz}, 3 \mathrm{H}), 0.83(\mathrm{~d}, J=6.7 \mathrm{~Hz}, 3 \mathrm{H}) ;{ }^{13} \mathrm{C}$ NMR $(100 \mathrm{MHz}$, $\left.\mathrm{CDCl}_{3}\right) \delta 193.8,193.2,167.4,161.3,159.4,156.5,149.8,139.1,133.4,131.7,130.4$ 130.2(9), 130.2(7), 129.3, 122.3, 122.1, 122.0, 118.4, 117.0, 116.4, 116.1, 94.6, 94.4, 91.2, 56.6, 56.2, 42.4, 24.1, 23.1; IR $v_{\max } 2959,1740,1702,1679,1607,1498,1389,1237,1152$, 1121, 1079, $989 \mathrm{~cm}^{-1}$; MS (ESI, +ve) $m / z 606\left[(\mathrm{M}+\mathrm{Na})^{+}, 90 \%\right], 584\left[(\mathrm{M}+\mathrm{H})^{+}, 100\right]$; HRMS $(\mathrm{ESI},+\mathrm{ve})$ Found: $(\mathrm{M}+\mathrm{Na})^{+}, 606.2107 . \mathrm{C}_{34} \mathrm{H}_{33} \mathrm{NNaO}_{8}$ requires $(\mathrm{M}+\mathrm{Na})^{+}, 606.2104$.

Potassium [1-(tert-Butoxycarbonyl)-1H-indol-3-yl]trifluoroborate (18). Following a procedure reported by Aggarwal, ${ }^{15}$ a magnetically stirred mixture of 3-Bpin- $N$-Boc-indole ${ }^{16}$ $(9.60 \mathrm{~g}, 28 \mathrm{mmol})$ in methanol/THF (90 mL of a 7:2 v/v mixture) was treated, dropwise at 0 ${ }^{\circ} \mathrm{C}$, with a solution of $\mathrm{KHF}_{2}(9.93 \mathrm{~g}, 126 \mathrm{mmol})$ in water $(33 \mathrm{~mL})$ and the ensuing white suspension was stirred at $20^{\circ} \mathrm{C}$ for $3 \mathrm{~h}$ then concentrated under reduced pressure. The residue thus obtained was re-dissolved in methanol/water $(50 \mathrm{~mL}$ of a $1: 1 \mathrm{v} / \mathrm{v}$ mixture) and all the volatile materials were again removed under reduced pressure. This evaporation-dissolution cycle was repeated a further four times and the white solid thereby obtained was treated with acetone $(100 \mathrm{~mL})$ and ensuing mixture then filtered (through filter paper) and the filtrate soobtained concentrated under reduced pressure. The resulting solid was then dried over $\mathrm{P}_{2} \mathrm{O}_{5}$ for $16 \mathrm{~h}$ to afford compound $\mathbf{1 8}^{17}$ (8.99 g, 99\%) as a colorless, amorphous solid. ${ }^{1} \mathrm{H}$ NMR $\left(400 \mathrm{MHz},\left(\mathrm{CD}_{3}\right)_{2} \mathrm{CO}\right) \delta 8.03(\mathrm{~d}, J=8.0 \mathrm{~Hz}, 1 \mathrm{H}), 7.78(\mathrm{~d}, J=8.0 \mathrm{~Hz}, 1 \mathrm{H}), 7.33(\mathrm{~s}, 1 \mathrm{H}), 7.11$ 
$(\mathrm{t}, J=8.0 \mathrm{~Hz}, 1 \mathrm{H}), 7.04(\mathrm{t}, J=8.0 \mathrm{~Hz}, 1 \mathrm{H}), 1.64(\mathrm{~s}, 9 \mathrm{H}) ;{ }^{13} \mathrm{C} \mathrm{NMR}\left[100 \mathrm{MHz},\left(\mathrm{CD}_{3}\right)_{2} \mathrm{CO}\right] \delta$ $150.7,137.0,136.6,127.7,123.8,122.9,121.9,114.8,82.5,28.2$ (one signal obscured or overlapping); ${ }^{11} \mathrm{~B}$ NMR $\left(128 \mathrm{MHz},\left(\mathrm{CD}_{3}\right)_{2} \mathrm{CO}\right) \delta 3.48 ;{ }^{19} \mathrm{~F} \mathrm{NMR}\left(376 \mathrm{MHz},\left(\mathrm{CD}_{3}\right)_{2} \mathrm{CO}\right) \delta$ $-138.3 ;$ IR $v_{\max } 2984,1724,1706,1455,1370,1250,1161,1127,1084,983,928,900,754$ $\mathrm{cm}^{-1}$; MS (ESI, +ve) m/z 362 [(M+K) $\left.)^{+}, 100 \%\right]$; HRMS (ESI, +ve) Found: $(\mathrm{M}+\mathrm{K})^{+}, 362.0340$. $\mathrm{C}_{13} \mathrm{H}_{14} \mathrm{BF}_{3} \mathrm{KNO}_{2}$ requires $(\mathrm{M}+\mathrm{K})^{+}, 362.0344$.

tert-Butyl 3-((1R)-1-(3a-Isobutyl-1,2-bis(4-(methoxymethoxy)phenyl)-3,5dioxo-3,3a-dihydro-5H-benzo[d]pyrrolo[2,1-b][1,3]oxazin-7-yl)-3-

oxopropyl)-1H-indole-1-carboxyl -ate (19). A magnetically stirred mixture of compound 17 (41 mg, $0.07 \mathrm{mmol}),(R)-3,3^{\prime}-\left(\mathrm{C}_{7} \mathrm{~F}_{7}\right)_{2}$-BINOL (25 mg, $\left.0.035 \mathrm{mmol}\right)$, potassium 1-(t-butyloxycarbonyl)-3-indoletrifluoroborate (18) $(68 \mathrm{mg}, 0.21 \mathrm{mmol})$ and molecular sieves (200 mg of $4 \AA$ powdered material) in dry toluene $(3 \mathrm{~mL})$ was heated at $80{ }^{\circ} \mathrm{C}$ in a sealed tube for $48 \mathrm{~h}$. The cooled reaction mixture was filtered through a pad of TLC-grade silica and the filtrate concentrated under reduced pressure. The residue thus obtained was subjected to flash chromatography (silica, 2:1 v/v hexane/ethyl acetate elution) to afford, after concentration of the appropriate fractions $\left(R_{f}=0.2\right.$ in $2: 1 \mathrm{v} / \mathrm{v}$ hexane/ethyl acetate), compound $19(45 \mathrm{mg}, 80 \%)$ as a 1:1 mixture of diastereoisomers and as a clear, light-yellow oil, $[\alpha]_{\mathrm{D}}{ }^{24}=+60\left(c 0.3, \mathrm{CHCl}_{3}\right) .{ }^{1} \mathrm{H}$ NMR $\left(400 \mathrm{MHz}, \mathrm{CDCl}_{3}\right) \delta 9.81(\mathrm{~s}, 1 \mathrm{H}), 8.19-8.03$ (complex m, 2H), 7.49 (s, 1H), 7.34-6.99 (complex m, 10H), 6.88 (m, 2H), $6.28(\mathrm{~m}, 1 \mathrm{H})$, 5.27-5.17 (complex m, 2H), $5.11(\mathrm{~m}, 2 \mathrm{H}), 4.80(\mathrm{~m}, 1 \mathrm{H}), 3.57-3.49$ (complex m, 3H), 3.46 (m, 3H), 3.42-3.07 (complex m, 2H), 2.32 (m, 1H), 2.00-1.87 (complex m, 1H), 1.77-1.66 (complex m, 10H), 0.96-0.90 (complex 3H), 0.84-0.77 (complex m, 3H); ${ }^{13} \mathrm{C}$ NMR (100 $\left.\mathrm{MHz}, \mathrm{CDCl}_{3}\right) \delta 199.9,199.8,194.1(0), 194.0(7), 168.5(3), 168.5(1), 161.9,161.8,159.1$, 
156.2, 149.7, 139.4(2), 139.3(6), 136.2, 136.1, 135.9, 134.7, 134.5, 130.6, 130.3, 130.2, 130.1, 129.7(1), 129.6(5), 129.2(0), 129.1(5), 124.9, 122.9, 122.8(4), 122.7(8), 122.6(2), 122.6(0), 122.4, 122.3, 122.2, 122.0, 121.7, 121.6, 119.3(3), 119.2(7), 118.2, 118.1, 116.8, 116.7, 116.0, 115.6(0), 115.5(7), 115.2(8), 115.2(7), 94.5(4), 94.5(2), 94.4, 91.3, 91.2, 84.2, $56.5,56.1,49.0,48.9,42.1,42.0,35.9,35.8,29.8,28.3,24.0,23.9,23.1 ;$ MS (ESI, +ve) $\mathrm{m} / \mathrm{z}$ $801\left[(\mathrm{M}+\mathrm{H})^{+}, 100 \%\right]$; HRMS (ESI, +ve) Found: $(\mathrm{M}+\mathrm{H})^{+}$, 801.3381. $\mathrm{C}_{47} \mathrm{H}_{49} \mathrm{~N}_{2} \mathrm{O}_{10}$ requires 801.3387.

tert-Butyl

3-((1R,2S)-2,3-Dihydroxy-1-(3a-isobutyl-1,2-bis(4(methoxymethoxy)phenyl)-3,5-dioxo-3,3a-dihydro-5H-benzo[d]pyrrolo[2,1b][1,3]oxazin-7-yl)propyl)- $1 \boldsymbol{H}$-indole-1-carboxylate (20). A magnetically stirred mixture of compound $19(67 \mathrm{mg}, 0.083 \mathrm{mmol})$ and nitrosobenzene $(8.9 \mathrm{mg}, 0.083 \mathrm{mmol})$ in acetonitrile $(0.5 \mathrm{~mL})$ was cooled to $4{ }^{\circ} \mathrm{C}$ then treated with $D$-proline $(2.9 \mathrm{mg}, 0.025 \mathrm{mmol})$. The ensuing and initially green-colored mixture was stirred at $4{ }^{\circ} \mathrm{C}$ for $6 \mathrm{~h}$ during which time the color of the mixture turned to yellow (and thus marking the end-point of the $\alpha$ aminoxylation reaction) and at which stage it was diluted with 1,2-dichloroethane $(2 \mathrm{~mL})$ and treated with $\mathrm{NaBH}(\mathrm{OAc})_{3}(145 \mathrm{mg}, 0.66 \mathrm{mmol})$. The resulting yellow suspension was stirred at $22{ }^{\circ} \mathrm{C}$ for $16 \mathrm{~h}$ then treated, successively, with $\mathrm{NaHCO}_{3}(2 \mathrm{~mL}$ of a saturated aqueous solution) and water $(10 \mathrm{~mL})$ before being extracted with ethyl acetate $(3 \times 10 \mathrm{~mL})$. The combined organic phases were washed with brine $(1 \times 30 \mathrm{~mL})$ then dried $\left(\mathrm{Na}_{2} \mathrm{SO}_{4}\right)$, filtered and concentrated under reduced pressure. The residue thus obtained was dissolved in dry dichloromethane $(2 \mathrm{~mL})$ and the resulting solution cooled to $4{ }^{\circ} \mathrm{C}$ before being treated with nitrosobenzene (17 $\mathrm{mg}, 0.16 \mathrm{mmol})$. The ensuing green-colored was mixture was stirred at 4 ${ }^{\circ} \mathrm{C}$ for $4 \mathrm{~h}$ and then subjected to flash chromatography (silica, 2:3 v/v hexane/ethyl acetate elution) to afford, after concentration of the appropriate fractions $\left(R_{f}=0.2\right.$ in $1: 1 \mathrm{v} / \mathrm{v}$ 
hexane/ethyl acetate), compound $\mathbf{2 0}(24.7 \mathrm{mg}, 36 \%)$ as a clear, yellow oil and a 1:1 mixture of diastereoisomers, $[\alpha]_{\mathrm{D}}{ }^{24}=-29\left(c 0.2, \mathrm{CHCl}_{3}\right) .{ }^{1} \mathrm{H} \mathrm{NMR}\left(600 \mathrm{MHz}, \mathrm{CD}_{3} \mathrm{OD}\right) \delta 8.13(\mathrm{~d}, J=$ $2.1 \mathrm{~Hz}, 0.5 \mathrm{H}), 8.11(\mathrm{~d}, J=2.1 \mathrm{~Hz}, 0.5 \mathrm{H}), 8.10(\mathrm{~m}, 1 \mathrm{H}), 7.77(\mathrm{~s}, 0.5 \mathrm{H}), 7.75(\mathrm{~s}, 0.5 \mathrm{H}), 7.51$ (m, 0.5H), $7.49(\mathrm{~m}, 0.5 \mathrm{H}), 7.38(\mathrm{~d}, J=7.9 \mathrm{~Hz}, 0.5 \mathrm{H}), 7.35(\mathrm{~d}, J=7.8 \mathrm{~Hz}, 0.5 \mathrm{H}), 7.26(\mathrm{~m}$, 1H), 7.19-7.12 (complex m, 3H), 7.08 (m, 1H), 7.05-7.01 (complex m, 3H), 6.86 (d, $J=8.9$ $\mathrm{Hz}, 1 \mathrm{H}), 6.85(\mathrm{~d}, J=8.9 \mathrm{~Hz}, 1 \mathrm{H}), 6.38(\mathrm{~d}, J=8.5 \mathrm{~Hz}, 0.5 \mathrm{H}), 6.36(\mathrm{~d}, J=8.5 \mathrm{~Hz}, 0.5 \mathrm{H}), 5.23$ (s, 1H), $5.19(\mathrm{~m}, 1 \mathrm{H}), 5.11(\mathrm{~s}, 2 \mathrm{H}), 4.42-4.36$ (complex m, 2H), 3.52-3.45 (complex m, 2H), $3.48(\mathrm{~s}, 1.5 \mathrm{H}), 3.44$ (s, 1.5H), $3.40(\mathrm{~s}, 3 \mathrm{H}), 2.22-2.14$ (complex m, 1H), 2.04-1.93 (complex m, 1H), 1.80-1.71 (complex m, 1H), $1.69(\mathrm{~s}, 4.5 \mathrm{H}), 1.68(\mathrm{~s}, 4.5 \mathrm{H}), 0.93(\mathrm{~d}, J=6.7 \mathrm{~Hz}$, $1.5 \mathrm{H}), 0.92(\mathrm{~d}, J=6.7 \mathrm{~Hz}, 1.5 \mathrm{H}), 0.82(\mathrm{~d}, J=6.7 \mathrm{~Hz}, 1.5 \mathrm{H}), 0.81(\mathrm{~d}, J=6.7 \mathrm{~Hz}, 1.5 \mathrm{H})$

(signals due to hydroxyl group protons not observed); ${ }^{13} \mathrm{C}$ NMR $\left(150 \mathrm{MHz}, \mathrm{CD}_{3} \mathrm{OD}\right) \delta$ 196.3(1), 196.2(9), 172.1(0), 172.0(9), 163.5, 163.4, 160.7, 160.6, 157.7, 151.1, 139.6(2), 139.5(7), 137.6, 137.5, 137.0, 132.5, 132.4, 131.6, 131.5, 131.4, 131.2, 125.6, 124.3, 124.1, 123.5(9), 123.5(7), 123.4(2), 123.3(8), 123.1, 122.9, 122.8, 122.7, 120.4, 120.3, 118.8(2), 118.7(9), 117.7(1), 117.6(7), 116.9, 116.2(0), 116.1(8), 116.1(5), 116.1, 95.5(0), 95.4(7), 95.4, 92.7, 92.6, 85.0, 74.4, 74.2, 65.6, 65.5, 56.5(9), 56.5(6), 56.2, 45.0, 43.0, 42.9, 28.4(3), 28.4(2), 25.2, 25.1, 24.2(1), 24.1(7), 23.5(1), 23.4(9); IR $v_{\max } 3449,2925,1735,1611,1499$, 1453, 1371, 1240, 1155, 1080, $996 \mathrm{~cm}^{-1}$; MS (ESI, +ve) $\mathrm{m} / z 841\left[(\mathrm{M}+\mathrm{Na})^{+}, 100 \%\right]$; HRMS Found: $(\mathrm{M}+\mathrm{Na})^{+}$841.3312. $\mathrm{C}_{47} \mathrm{H}_{50} \mathrm{~N}_{2} \mathrm{O}_{11}$ requires 841.3312.

7-((1R,2S)-2,3-Dihydroxy-1-(1H-indol-3-yl)propyl)-1,2-bis(4-hydroxyphenyl)-3a-isobutyl-5H-benzo[d]pyrrolo[2,1-b][1,3]oxazine-3,5(3aH)-dione (Discoipyrrole $\quad$ D, 4). A magnetically stirred solution of compound $20(24.5 \mathrm{mg}, 0.03 \mathrm{mmol})$ in dichloromethane (2 $\mathrm{mL})$ was treated with trifluoroacetic acid $(460 \mu \mathrm{L}, 6 \mathrm{mmol})$ and the ensuing brown-colored 
reaction mixture was stirred at $22{ }^{\circ} \mathrm{C}$ for $2 \mathrm{~h}$ before being treated, successively, with $\mathrm{NaHCO}_{3}(1 \times 2 \mathrm{~mL}$ of a saturated aqueous solution $)$ and water $(1 \times 10 \mathrm{~mL})$ and then extracted with ethyl acetate $(3 \times 10 \mathrm{~mL})$. The combined organic phases were washed with brine $(1 \times 30 \mathrm{~mL})$ then dried $\left(\mathrm{Na}_{2} \mathrm{SO}_{4}\right)$, filtered, and concentrated under reduced pressure. The residue thus obtained was dissolved in THF $(2 \mathrm{~mL})$, the resulting solution treated with $\mathrm{HCl}\left(2 \mathrm{~mL}\right.$ of a $4.0 \mathrm{M}$ aqueous solution) and the mixture thus obtained stirred at $22{ }^{\circ} \mathrm{C}$ for 3 h before being diluted with $\mathrm{NaHCO}_{3}$ (2 $\mathrm{mL}$ of a saturated aqueous solution) and water (10 $\mathrm{mL})$ then extracted with ethyl acetate $(3 \times 10 \mathrm{~mL})$. The combined organic phases were washed with brine $(1 \times 30 \mathrm{~mL})$ then dried $\left(\mathrm{Na}_{2} \mathrm{SO}_{4}\right)$, filtered and concentrated under reduced pressure. The yellow oil thus obtained was subjected to flash chromatography (silica, 12:1 $\mathrm{v} / \mathrm{v}$ ethyl acetate/isopropanol elution) and thus affording, after concentration of the appropriate fractions $\left(R_{f}=0.6\right)$, compound $4(11 \mathrm{mg}, 60 \%)$ as a clear, light-yellow oil and a 1:1 mixture of diastereoisomers, $[\alpha]_{\mathrm{D}}{ }^{25}=+25(c 0.2, \mathrm{MeOH}) .{ }^{1} \mathrm{H} \mathrm{NMR}\left(800 \mathrm{MHz}, \mathrm{CD}_{3} \mathrm{OD}\right)$ $\delta 8.13(\mathrm{~d}, J=2.1 \mathrm{~Hz}, 0.5 \mathrm{H}), 8.10(\mathrm{~d}, J=2.1 \mathrm{~Hz}, 0.5 \mathrm{H}), 7.52(\mathrm{td}, J=8.8$ and $2.1 \mathrm{~Hz}, 1 \mathrm{H})$, $7.42(\mathrm{~d}, J=8.0 \mathrm{~Hz}, 0.5 \mathrm{H}), 7.40(\mathrm{~d}, J=8.0 \mathrm{~Hz}, 0.5 \mathrm{H}), 7.33(\mathrm{~d}, J=8.1 \mathrm{~Hz}, 0.5 \mathrm{H}), 7.32(\mathrm{~d}, J=$ $8.1 \mathrm{~Hz}, 0.5 \mathrm{H}), 7.31(\mathrm{~s}, 0.5 \mathrm{H}), 7.30(\mathrm{~s}, 0.5 \mathrm{H}), 7.07(\mathrm{~m}, 1 \mathrm{H}), 7.05-7.02$ (complex m, 2H), 6.97-6.90 (complex m, 3H), $6.82(\mathrm{~d}, J=8.9 \mathrm{~Hz}, 1 \mathrm{H}), 6.80(\mathrm{~d}, J=8.8 \mathrm{~Hz}, 1 \mathrm{H}), 6.62(\mathrm{dm}, J=$ $8.8 \mathrm{~Hz}, 1 \mathrm{H}), 6.61(\mathrm{dm}, J=8.8 \mathrm{~Hz}, 1 \mathrm{H}), 6.39(\mathrm{~d}, J=8.5 \mathrm{~Hz}, 0.5 \mathrm{H}), 6.38(\mathrm{~d}, J=8.5 \mathrm{~Hz}, 0.5 \mathrm{H})$, 4.43-4.40 (complex m, 1H), $4.36(\mathrm{~d}, J=8.1 \mathrm{~Hz}, 0.5 \mathrm{H}), 4.35(\mathrm{~d}, J=8.1 \mathrm{~Hz}, 0.5 \mathrm{H}), 3.56(\mathrm{~m}$, 1H), 3.45 (complex m, 1H), $2.15(\mathrm{~m}, 1 \mathrm{H}), 1.99(\mathrm{~m}, 0.5 \mathrm{H}), 1.95(\mathrm{~m}, 0.5 \mathrm{H}), 1.73(\mathrm{~m}, 1 \mathrm{H})$, $0.91(\mathrm{t}, J=6.4 \mathrm{~Hz}, 3 \mathrm{H}), 0.80(\mathrm{~d}, J=6.7 \mathrm{~Hz}, 3 \mathrm{H})$ (signals due to $\mathrm{OH}$ and $\mathrm{NH}$ group protons not observed); ${ }^{13} \mathrm{C}$ NMR (200 MHz, $\left.\mathrm{CD}_{3} \mathrm{OD}\right) \delta 196.6,196.5,172.4(3), 172.4(1), 163.8$, 161.1, 157.6, 141.8(3), 141.8(1), 137.9(8), 137.9(6), 137.3, 137.2, 136.5, 132.0, 131.9, 131.6, 
131.4, 128.0, 123.3(2), 123.3(1), 122.9(2), 122.8(6), 122.5(9), 122.5(8), 121.8, 121.1, 119.8(3), 119.8(2), 119.5(4), 119.5(0), 118.6, 118.5, 116.8(9), 116.8(7), 116.8, 116.7, 116.1, 116.0, 115.9, 112.4, 112.3, 92.6(3), 92.6(2), 75.3, 75.1, 66.1, 66.0, 46.1(2), 46.0(9), 42.9(6), 42.9(5), 25.2, 24.2(2), 24.1(9), 23.4(2), 23.4(0) (nineteen signals obscured or overlapping);; IR $v_{\max } 3364,2959,2927,1720,1612,1498,1387,1273,1240,1172,1070,1042 \mathrm{~cm}^{-1}$; MS $(\mathrm{ESI},+\mathrm{ve}) \mathrm{m} / z 653\left[(\mathrm{M}+\mathrm{Na})^{+}, 40 \%\right], 631\left[(\mathrm{M}+\mathrm{H})^{+}, 100\right]$; HRMS Found: $(\mathrm{M}+\mathrm{H})^{+} 631.2441$. $\mathrm{C}_{38} \mathrm{H}_{35} \mathrm{~N}_{2} \mathrm{O}_{7}$ requires 631.2444 .

\section{ASSOCIATED CONTENT}

\section{Supporting Information}

The Supporting Information is available free of charge on the ACS Publications website at DOI: 10.1021/acs.joc.XXXXXXX. Tabular comparison of the ${ }^{13} \mathrm{C}$ NMR data reported for discoipyrrole D with those recorded on the synthetically derived compound $4 .{ }^{1} \mathrm{H}$ and ${ }^{13} \mathrm{C}$ NMR spectra of compounds 7-9, 11-20 and 4 (PDF).

\section{AUTHOR INFORMATION}

Corresponding Author

*E-mail: Martin.Banwell@anu.edu.au.

Notes

The authors declare no competing financial interest.

\section{ACKNOWLEDGEMENTS}


We thank the Australian Research Council for financial support. Y.Z. is the grateful recipient of a PhD Scholarship provided by the China Scholarship Council of the People's Republic of China.

\section{REFERENCES}

1. Hu, Y.; Potts, M. B.; Colosimo, D.; Herrera-Herrera, M. L.; Legako, A. G.; Yousufuddin, M.; White, M. A.; MacMillan, J. B. J. Am. Chem. Soc. 2013, 135, 13387.

2. Colosimo, D. A.; MacMillan, J. B. J. Am. Chem. Soc. 2016, 138, 2383.

3. Shih, J.-L.; Nguyen, T. S.; May, J. A. Angew. Chem. Int. Ed. 2015, 54, 9931.

4. Zhang, Y.; Banwell, M. G.; Carr, P. D.; Willis, A. C. Org. Lett. 2016, 18, 704.

5. Altman, R. A.; Buchwald, S. L. Nat. Protoc. 2007, 2, 2474.

6. Almond-Thynne, J.; Blakemore, D. C.; Pryde, D. C.; Spivey, A. C. Chem. Sci., 2017, 8,40 and references cited therein.

7. Vedejs, E.; Larsen, S. Org. Synth. 1985, 64, 127.

8. The pathway by which this type of oxidative cyclization reaction takes is currently being explored in our laboratories.

9. Banwell, M. G.; Zhang, Y. Modular Synthesis of Discoipyrrole Type Alkaloids and Analogues. PCT/AU2016/000397 (filed 13/12/16)

10. Tasneem, Synlett 2003, 138.

11. Schulz, C. M.; Lehmann, L.; Blatrix, R.; Jaisson, P.; Hefetz, A.; Francke, W. J. Chem. Ecol. 2002, 28, 2541.

12. Zhong, G. Angew. Chem. Int. Ed. 2003, 42, 4247. 
13. Still, W. C.; Kahn, M.; Mitra, A. J. Org. Chem. 1978, 43, 2923.

14. Pangborn, A. B.; Giardello, M. A.; Grubbs, R. H.; Rosen, R. K.; Timmers, F. J. Organometallics 1996, 15, 1518.

15. Bagutski, V.; Ros, A.; Aggarwal, V. K. Tetrahedron, 2009, 65, 9956.

16. Johansson Seechurn, C. C. C.; Sivakumar, V.; Satoskar, D.; Colacot, T. J. Organometallics, 2014, 33, 3514.

17. Berionni, G.; Mayer, P.; Mayr, H. Acta Cryst. 2012, E68, m551. 